\title{
A!
}

This is an electronic reprint of the original article.

This reprint may differ from the original in pagination and typographic detail.

Dmitriev, V.V.; Kosarev, I.V.; Krusius, M.; Ponarin, D.V.; Ruutu, V.M.H.; Volovik, G.E.

\section{Stable spin precession at one half of equilibrium magnetization in superfluid $3 \mathrm{He}-\mathrm{B}$}

Published in:

Physical Review Letters

DOI:

10.1103/PhysRevLett.78.86

Published: 01/01/1997

Document Version

Publisher's PDF, also known as Version of record

Please cite the original version:

Dmitriev, V. V., Kosarev, I. V., Krusius, M., Ponarin, D. V., Ruutu, V. M. H., \& Volovik, G. E. (1997). Stable spin precession at one half of equilibrium magnetization in superfluid 3He-B. Physical Review Letters, 78(1), 86-89. https://doi.org/10.1103/PhysRevLett.78.86

This material is protected by copyright and other intellectual property rights, and duplication or sale of all or part of any of the repository collections is not permitted, except that material may be duplicated by you for your research use or educational purposes in electronic or print form. You must obtain permission for any other use. Electronic or print copies may not be offered, whether for sale or otherwise to anyone who is not an authorised user. 


\title{
Stable Spin Precession at One Half of Equilibrium Magnetization in Superfluid ${ }^{3} \mathrm{He}-B$
}

\author{
V. V. Dmitriev, ${ }^{1,2}$ I. V. Kosarev, ${ }^{1}$ M. Krusius, ${ }^{2}$ D. V. Ponarin, ${ }^{1}$ \\ V.M. H. Ruutu, ${ }^{2}$ and G. E. Volovik ${ }^{2,3}$ \\ ${ }^{1}$ Kapitza Institute for Physical Problems, 117334 Moscow, Russia \\ ${ }^{2}$ Low Temperature Laboratory, Helsinki University of Technology, 02150 Espoo, Finland \\ ${ }^{3}$ Landau Institute for Theoretical Physics, 117334 Moscow, Russia \\ (Received 10 June 1996; revised manuscript received 11 October 1996)
}

\begin{abstract}
New modes of spin precession have been observed in superfluid ${ }^{3} \mathrm{He}-B$ with a precessing magnetization $M=p M_{\mathrm{eq}}$, where $p=1, \frac{1}{2}$, and $\approx 0$. These dynamic order parameter states are the first examples of stable precession with a magnetization different from the equilibrium value $M_{\text {eq }}$. The $p=\frac{1}{2}$ mode is the second example of a phase-coherent state of a spin superfluid. The new states can be excited close to $T_{c}$ in the range $1-T / T_{c} \lesssim 0.02$ where dissipation is low and the energy barriers between the different local minima of the spin-orbit energy are small. [S0031-9007(96)01929-1]
\end{abstract}

PACS numbers: 67.57.Lm, 76.60.-k

A number of stable states of coherent spin precession have been observed and identified in NMR experiments in the normal and $B$ phases of liquid ${ }^{3} \mathrm{He}$ and in ${ }^{3} \mathrm{He}-{ }^{4} \mathrm{He}$ solutions [1-4]. Some of these states in ${ }^{3} \mathrm{He}-B$ display the same features of spontaneously broken symmetry that characterize quantum coherence in superfluids. Here the role of the supercurrent is played by the spin current. The main difference from a superfluid with a mass supercurrent is that in general the spin is not conserved. To stabilize a steady precessing state an external radiofrequency (rf) field has to be applied to compensate for spin relaxation. The smaller the dissipation, the smaller the rf field can be, and the closer the system is to the ideal coherent dynamic state of a spin superfluid.

In ${ }^{3} \mathrm{He}-B$ one such state is the homogeneously precessing domain (HPD) [1]. The spin-superfluid properties of the HPD are well known: its spin current displays the Josephson effect and it supports topological spin-current vortices and collective modes, which result from the spin rigidity of this coherent state $[5,6]$. In the HPD mode the magnetization $\mathbf{M}=\gamma \mathbf{S}$ ( $\mathbf{S}$ is the spin density and $\gamma$ the gyromagnetic ratio) precesses with the thermal equilibrium magnitude $\gamma S_{\mathrm{eq}}=\chi H$, where $\chi$ is the magnetic susceptibility, and $H$ the static magnetic field.

We report the observation of new precessing states in ${ }^{3} \mathrm{He}-B$. As opposed to the HPD, these states may have a fractional value of spin density $S=p S_{\mathrm{eq}}$ and can be stabilized with continuous wave (cw) NMR close to the superfluid transition temperature $T_{c}$. In particular the half-magnetization (HM) states with $p=\frac{1}{2}$ should display the generic properties of a coherently precessing spin state if they will be stabilized well below $T_{c}$.

Experiments. - NMR measurements were carried out with nuclear demagnetization cryostats both in Helsinki and Moscow in cylindrical sample containers. In Helsinki the cell was from quartz with diameter $D=5 \mathrm{~mm}$ and length $L=7 \mathrm{~mm}$ while in Moscow the inside surface was covered with lavsan polyethylene foil (similar to mylar) and the dimensions were $D=3.7 \mathrm{~mm}$ and $L=$ $4 \mathrm{~mm}$. The measurements were performed at pressures $P=0-12$ bars in magnetic fields $H=73-284$ Oe (or Larmor frequencies $\omega_{L} / 2 \pi=\gamma H / 2 \pi=235-920 \mathrm{kHz}$ ), oriented parallel to the container axis. With cw NMR we monitor the in-phase dispersion $\left(\propto S_{x}=S_{\perp} \cos \phi\right)$ and out-of-phase absorption $\left(\propto S_{y}=S_{\perp} \sin \phi\right)$ signals. In Moscow pulsed NMR measurements were also performed and the field homogeneity was improved from $\delta H / H \sim$ $7 \times 10^{-4}$ in Helsinki to $5 \times 10^{-5}$. It was then found that a small applied field gradient did not change the results qualitatively.

In a large transverse rf field $H_{\mathrm{rf}} \gtrsim 0.01$ Oe the cw NMR line shape changes drastically at temperatures $T \gtrsim 0.98 T_{c}$. In Fig. 1 the absorption and dispersion signals are shown, measured at fixed excitation frequency $\omega_{\text {rf }}$ during a slow sweep of $H$. The signals display discontinuities, which indicate transitions between different states of spin precession. The new states appear when the field is swept through resonance and the tipping angle $\beta$ of the precessing magnetization is large. A different sequence of states is traversed when the sweep is towards increasing (Fig. 1) or decreasing field (Fig. 2). The transitions are mostly of first order: they are hysteretic with respect to the sweep direction. In Fig. 2 the signals have been recorded during a slow warm-up. The lowermost spectrum is outside the temperature region of the new states and represents the usual NMR mode, when spin waves are excited in the static texture of the order parameter. We call this the spin wave (SW) state [7]. At higher temperatures new states appear. To identify these we perform a combination of $\mathrm{cW}$ and pulsed NMR measurement to determine the value of the precessing magnetization. By measuring the $\mathrm{cw}$ absorption and dispersion spectra we also record the transverse component of the spin density $S_{\perp}=S \sin \beta=\left(S_{x}^{2}+S_{y}^{2}\right)^{1 / 2}$ as a function of $T, H_{\mathrm{rf}}$, and the frequency shift $\omega_{\text {rf }}-\omega_{L}$. The results are compared with analytical solutions or numerical simulation. In 

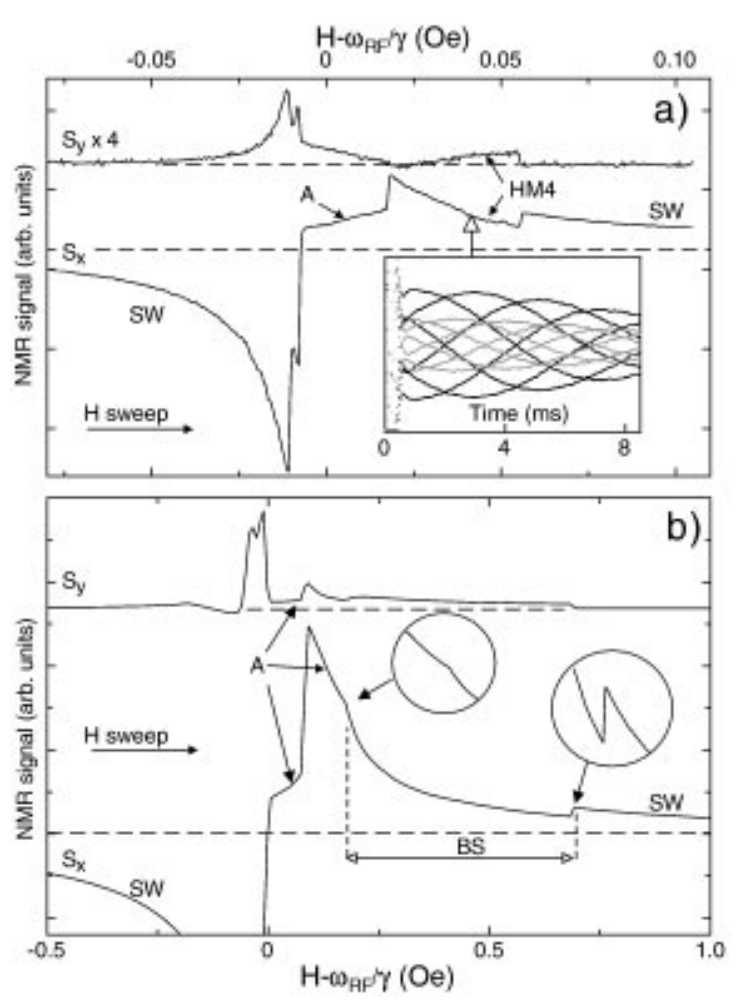

FIG. 1. Inset: Free induction decay signals of the HM4 state (light lines) and normal ${ }^{3} \mathrm{He}$ (dark lines), as recorded with a digital oscilloscope. The initial ratio of amplitudes is 0.52. Main frame: Measured absorption $\left(S_{y}\right)$ and dispersion $\left(S_{x}\right)$ during a sweep of increasing field. (a) Lavsan-coated cylinder, $P=0, \quad T \sim 0.997 T_{c}, \quad H_{\mathrm{rf}} \sim 0.02 \mathrm{Oe}, \omega_{\mathrm{rf}} / 2 \pi=$ $334 \mathrm{kHz}$. (b) Quartz cylinder, $P=5$ bars, $T \sim 0.990 T_{c}$, $H_{\text {rf }}=0.038 \mathrm{Oe} ; \omega_{\text {rf }} / 2 \pi=380 \mathrm{kHz}$. Unidentified features are marked with $A$. The free induction decay (FID) signal of HM4 in the inset was measured at the value of $H$ marked with the open arrow. The vertical scales are the same in Figs. 1(a) and 2; the dashed horizontal lines mark roughly zero signal level.

the latter we use a spatially homogeneous $H$, realistic values for the experimental parameters, and as dissipation the Leggett-Takagi relaxation [7].

Precessing states. - A number of stable solutions have been proposed to Leggett's equations of ${ }^{3} \mathrm{He}-B$ spin dynamics [8-12]. The relevant cases are classified in Fig. 3 in terms of the magnitude and direction of $\mathbf{S}$ in the precessing frame and the direction the orbital momentum density $\mathbf{L}$ in the laboratory frame. The conventional NMR mode, the SW state, corresponds to small oscillations of $\mathbf{S}$ about $\mathbf{S}_{\mathrm{eq}}$. In this state $\mathbf{L}$ forms a broad spatial texture due to the boundary conditions [7].

The existence of additional stable states is due to the particular form of the spin-orbit (dipole) interaction. After averaging over the fast Larmor precession, the dipole energy $F_{D}$ depends on the orientations of $\mathbf{S}$ and $\mathbf{L}$. It has a local minimum in the so-called resonance case, when $S \approx$ $S_{\text {eq. }}$. The SW and HPD states are examples of this resonance [13]. Additional resonances have been predicted to

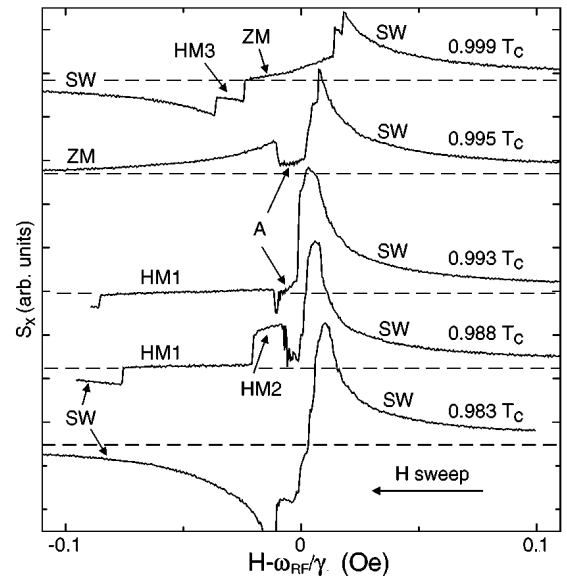

FIG. 2. Dispersion $\left(S_{x}\right)$ measured during a sweep of decreasing field and slow warm-up: lavsan-coated cell, $P=0$, $H_{\text {rf }} \sim 0.02 \mathrm{Oe} ; \omega_{\mathrm{rf}} / 2 \pi=461 \mathrm{kHz}$. The dotted curve represents a sweep of increasing field, starting from the SW state.

exist when $S \approx p S_{\text {eq }}$, where $p \approx 0$ [9], $\frac{1}{2}$, or 2 [10]. In Fig. 3, the stable precessing states should be located near these attractors in the $\mathbf{S}-\mathbf{L}$ space.

The stable states can be pictured as a double resonance. In the frame rotating with the frequency $\omega_{\mathrm{rf}}$, the rf field is constant and the total field is

$$
\tilde{\mathbf{H}}=\hat{\mathbf{z}}\left(\omega_{L}-\omega_{\mathrm{rf}}\right) / \gamma+\hat{\mathbf{x}} H_{\mathrm{rf}} .
$$

The doubly precessing states are stabilized by the dipole torque, which generates a resonance at the frequency $\tilde{\omega}_{L}=\gamma \tilde{H}$ in the rotating frame, if the condition

$$
S=\left(\chi / \gamma^{2}\right)\left(p \omega_{\mathrm{rf}}+\gamma \tilde{H}\right),
$$

is satisfied with $p=0, \frac{1}{2}$, 1 , or 2 . Magnetic relaxation and spatial inhomogeneity influence the resonances, but

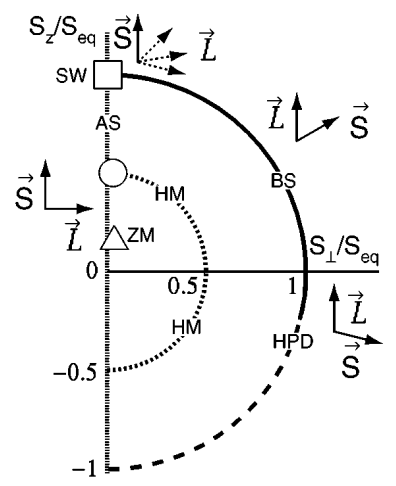

FIG. 3. Precessing states in the plane $S_{z} / S_{\text {eq }}-S_{\perp} / S_{\text {eq }}$. SW ( $\square$ ) denotes conventional NMR at low rf level. The BrinkmanSmith (BS) mode exists on the semicircle $S=S_{\text {eq }}$ (thick black line), with $\mathbf{L} \| \mathbf{H}$, when $\beta<104^{\circ}$. The phase-coherent HPD mode is the dashed continuation of this semicircle when $\beta \geqslant$ $104^{\circ}$. The half-magnetization (HM) mode (dashed semicircle) has $\frac{1}{2} S_{\text {eq }}$ while nearly zero magnetization (ZM) is observed in the ZM mode. The observed ZM $(\triangle)$ and HM $(\bigcirc)$ states are close to the line of aligned spins (AS) with $\mathbf{S} \| \mathbf{H} \perp \mathbf{L}$. 
do not essentially change the states except in the case of $p=0$, when the magnitude of $S$ is not determined by Eq. (2) but mainly by dissipation [9].

Half-magnetization modes. - We observe four NMR signatures which we ascribe to $p=\frac{1}{2}$ states: HM1, HM2, and HM3 appear at positive frequency shifts $\omega_{\text {rf }}>\omega_{L}$ (Fig. 2) and HM4 at negative shifts $\omega_{\text {rf }}<\omega_{L}$ (Fig. 1). Their identification as $p=\frac{1}{2}$ states is based on a measurement of the magnetization. At fixed $H$ in the desired state we switch off the rf pumping. Immediately after that a $90^{\circ}$ tipping pulse is applied and the FID signal is recorded (inset in Fig. 1). On comparing its amplitude with that of the FID obtained after a single $90^{\circ}$ tipping pulse in normal ${ }^{3} \mathrm{He}$ we find that for all $\mathrm{HM}$ modes the amplitude ratio is $0.50 \pm 0.03$ and not dependent on the phase of the $\mathrm{rf}$ pulse. This means that before the pulse the magnetization is roughly along $\mathbf{H}$ and $S \approx \frac{1}{2} S_{\mathrm{eq}}$. For all $\mathrm{HM}$ modes the FID duration is nearly the same as in normal ${ }^{3} \mathrm{He}$. This implies that, when $H_{\mathrm{rf}}=0$, they all reduce to the same HM state which preserves its configuration during the FID.

These features of the HM1, HM3, and HM4 states are well reproduced in numerical simulation. It also shows that in the presence of the rf field they share similar properties: (i) They lie near the positive intersection of the $p=\frac{1}{2}$ circle with the vertical line in Fig. 3. (ii) There is a degeneracy in the orientation of the orbital momentum: $\mathbf{L} \perp \mathbf{H}$. (iii) The spin precesses with $\omega_{\text {rf }}$ while the order parameter axis $\hat{\mathbf{n}}$ precesses with $\frac{1}{2} \omega_{\text {rf }}$ (applies to aligned HM states in general). By minimizing the dipole and spectroscopic energies, $F=F_{D}\left(s_{z}, l_{z}\right)-\left(\omega_{L}-\omega_{\mathrm{rf}}\right) S_{z}-S_{x} H_{\mathrm{rf}} / \gamma$, an analytic description can be derived for HM4 with $\omega_{\mathrm{rf}}<$ $\omega_{L}$, where [10]

$$
\begin{aligned}
F_{D}\left(s_{z}, l_{z}\right)= & \left(\chi / 10 \gamma^{2}\right) \Omega_{B}^{2} \\
& \times\left[1+2 l_{z}^{2} s_{z}^{2}+\left(1-l_{z}^{2}\right)\left(1-s_{z}^{2}\right)-(2 / 3)\right. \\
& \left.\times\left(1+l_{z}\right)\left(1+s_{z}\right) \sqrt{\left(1-l_{z}^{2}\right)\left(1-s_{z}^{2}\right)}\right] .
\end{aligned}
$$

Here $s_{z}=S_{z} / S, l_{z}=L_{z} / L$, and $\hat{\mathbf{z}} \| \mathbf{H} . \quad \Omega_{B}$ is the Leggett frequency of ${ }^{3} \mathrm{He}-B$ [7]. At relatively large negative frequency shift the two local minima from Ref. [10] merge into one with

$\sin \beta \approx\left(1+w^{2} / 4\right)^{-1 / 2}, \quad w=15 \omega_{\mathrm{rf}}\left(\omega_{\mathrm{rf}}-\omega_{L}\right) / 2 \Omega_{B}^{2}$.

For $T / T_{c}>0.995$ and $H-\omega_{\mathrm{rf}} / \gamma>0.05$ Oe one has $\beta \ll 1$, in agreement with our observation. Well below $T_{c}$ this state should have similar coherent spin-superfluid properties as the HPD.

At positive frequency shifts the HM3 state appears as a saddle point of $F$ in a narrow range of the field sweep where the phase of precession $\phi \approx \pi$ and $S_{x}<0$ (Fig. 2). Numerically HM1 and HM3 are readily identified while HM2 is not. The small dispersion of HM1 in a wide region of field sweep is similar to that found numeri-

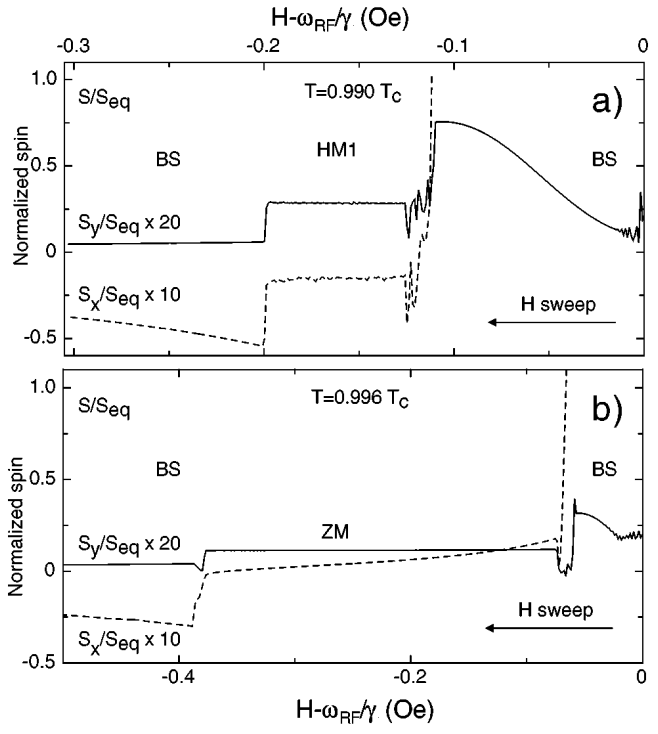

FIG. 4. Dispersion $\left(S_{x}\right)$, absorption $\left(S_{y}\right)$, and total spin $(S)$, normalized to $S_{\text {eq }}$, from numerical simulation at two temperatures. The sweep towards decreasing field was started from $\gamma H>\omega_{\text {rf }}$, but new states appear when $\gamma H<\omega_{\text {rf }}$. The parameters are $P=0, H_{\mathrm{rf}}=0.02 \mathrm{Oe}, \omega_{\mathrm{rf}} / 2 \pi=460 \mathrm{kHz}$, $T_{1}=0.1 \mathrm{~s}$. The same calculation gives for a sweep towards increasing field, starting from $\gamma H<\omega_{\text {rf }}$, the HM4 state, when $\gamma H>\omega_{\text {rf }}$, as seen in Fig. 1. Only spatially homogeneous order parameter states are considered in the simulation. Therefore the BS mode (or its mirror state at $\omega_{\mathrm{rf}}>\gamma H$ [12]) appears here outside the region of the HM and ZM states.

cally in Fig. 4(a), but the two ranges of frequency shift are different. Such inconsistency may be due to spatial inhomogeneity which is not included in the simulation. For instance the field inhomogeneity $(\delta H \sim 0.02$ Oe in Fig. 2) becomes especially important when $H-\omega_{\text {rf }} / \gamma \sim$ $\delta H$. In this case the sign of the frequency shift may vary within the sample and the precessing state may be spatially inhomogeneous. This may also be responsible for the unidentified features marked with $A$ in Figs. 1 and 2.

Zero-magnetization mode.-The signature of the $p \approx$ 0 state at positive frequency shift $\omega_{\mathrm{rf}}>\omega_{L}$ is shown in Fig. 2. The identification of this state is again based on pulsed NMR measurement of the magnitude of $S$. From the ratio of the initial FID amplitudes one can conclude that in the ZM state the spin decreases from $\lesssim 0.3 S_{\text {eq }}$ at small frequency shifts down to the noise level $\sim 0.02 S_{\text {eq }}$ at larger shifts. At large frequency shifts the transverse spin is found to be $S_{\perp} \propto T_{c}-T$ and it does not depend on $H_{\mathrm{rf}}$. Also the FID amplitude is found to vary with time such that we estimate $\beta \approx 20^{\circ}-30^{\circ}$. All these features are reproduced in the numerical simulation. It furthermore shows that the relaxation of the magnitude of $S$ after switching off the rf pumping is sufficiently slow so that pulsed NMR can be used for estimating $S$ and that the ZM state remains stable at small negative frequency shifts [H- $\omega_{\text {rf }} / \gamma \leqslant 0.1$ Oe for the conditions of Fig. 4(b)]. Thus the region marked by $A$ in Fig. 1(a) might also 
correspond to the ZM state. In Ref. [9] a state with $p \approx 0$ has been discussed analytically. There $\mathbf{L}$ was assumed to be $\| \mathbf{H}$, but the simulation shows that $\mathbf{L}$ prefers to be $\perp \mathbf{H}$.

Brinkman-Smith mode. - In the HPD mode spin currents maintain phase coherence of the spin precession and $\beta \gtrsim \theta_{L}$, where $\theta_{L} \approx 104^{\circ}$ is the Leggett angle [7]. The precursor of the HPD, the Brinkman-Smith state (BS) [8], with $\beta<\theta_{L}$, was actually the first of the extraodinary precessing states which was observed in early pulsed NMR experiments [14]. Here we find for the first time that the BS mode can be stabilized in cw NMR [Fig. 1(b)]. In this case $\mathbf{S}$ is oriented along $\tilde{\mathbf{H}}$ in the rotating frame $[11,12], \mathbf{S} \approx S_{\mathrm{eq}} \tilde{\mathbf{H}} / \tilde{H}$ and $\sin \beta=H_{\mathrm{rf}} / \tilde{H}$. Experimentally the BS mode is identified by its transverse spin density $S_{\perp}=S_{\mathrm{eq}} \sin \beta$, which was found to be in perfect agreement with the prediction.

A transition between any two states in Fig. 3, which involves a reorientation of $\mathbf{L}$, corresponds to a textural transformation. In the BS state the dipole energy orients $\mathbf{L}$ along $\mathbf{H}$ and the $\mathrm{SW} \rightarrow \mathrm{BS}$ or $\mathrm{BS} \rightarrow \mathrm{SW}$ transitions are to be accompanied by first order textural transformations. An anomaly observed in pulsed NMR measurements in Ref. [15] is explained by the SW $\rightarrow$ BS transition, as proposed in Ref. [16]. We observe the opposite case $\mathrm{BS} \rightarrow \mathrm{SW}$ [Fig. 1(b)] while the SW $\rightarrow$ BS transition is obscured by unidentified features at a small frequency shift where the SW mode becomes unstable.

There are differences in the results measured with the two sample cells. The ZM mode has been observed in both cylinders, but the HM modes were observed only in the lavsan-coated cell while the BS state was stable only in the quartz cell. This may be caused by the cylinder dimensions, field inhomogeneity, or relaxation at the walls. The effective longitudinal relaxation time $T_{1}$ was measured to be $1 \mathrm{~min}$ in the quartz and $1 \mathrm{~s}$ in the lavsan cells at $T \gtrsim$ $T_{c}(P=0)$. In the simulation it is lumped into an effective bulk $T_{1}$. The stable ranges of the new states were found to be sensitive to the choice of $T_{1}$. A further possibility is that a textural transformation to the BS state triggers a transition to the HM or ZM states in the lavsan container. It has a smaller volume by a factor of 5 and perhaps the latter modes can more readily be created uniformly in this case.

In conclusion, we have observed the first examples of stable dynamic spin modes with a well-defined precessing magnetization which differs from the thermal equilibrium value. The existence of such modes had been proposed before theoretically $[9,10]$, but it was not known in what conditions they might be stabilized. It is now clear that they appear after crossing the resonance at $\omega_{\mathrm{rf}}=\omega_{L}$ at a high rf level so that $\mathbf{S}$ is deflected by a large amount $\left(\beta \sim 180^{\circ}\right)$. Simultaneously the HPD mode with $\mathbf{L} \| \mathbf{H}$ should be unstable towards a reorientation to $\mathbf{L} \perp \mathbf{H}$. Well below $T_{c}$ this instability is difficult to reach due to the dipole torque and Leggett-Takagi dissipation, which rapidly grow with increasing $\beta$ above $\theta_{L}$. Numerical simulation of the resonance conditions from Leggett's spin dynamic equations also confirms that the new states can be reached in the vicinity of $T_{c}$ where $F_{D} \propto T_{c}-T$ is small and the more conventional modes with $S=S_{\text {eq }}$ become unstable already with tipping angles $\beta<180^{\circ}$ and, in fact, with $\beta$ as small as $\sim 140^{\circ}$.

[1] A. Borovik-Romanov, Yu. Bunkov, V. Dmitriev, and Yu. Mukharsky, JETP Lett. 40, 1033 (1984); I. Fomin, ibid. 40, 1037 (1984).

[2] Yu. Bunkov, S. Fisher, A. Guénault, G. Pickett, and S. Zakazov, Physica (Amsterdam) 194B, 827 (1994).

[3] G. Nunes, C. Jin, D. Hawthorne, A. Putnam, and D. Lee, Phys. Rev. B 46, 9082 (1992); H. Akimoto, O. Ishikawa, G. Oh, M. Nakagawa, T. Hata, and T. Kodama, J. Low Temp. Phys. 82, 295 (1991).

[4] V. Dmitriev and I. Fomin, JETP Lett. 59, 378 (1994); V. Dmitriev, V. Moroz, and S. Zakazov, J. Low Temp. Phys. 101, 141 (1995).

[5] I. Fomin, Physica (Amsterdam) 169B, 153 (1991).

[6] Yu. Bunkov, in Progress in Low Temperature Physics, edited by W. Halperin (Elsevier, Amsterdam, 1995), Vol. 14, p. 69.

[7] D. Vollhardt and P. Wölfle, The Superfluid Phases of ${ }^{3} \mathrm{He}$ (Taylor \& Francis, London, 1990).

[8] W. Brinkman and H. Smith, Phys. Lett. 53A, 43 (1975).

[9] E. Sonin, Sov. Phys. JETP 67, 1791 (1988).

[10] G. Kharadze and G. Vachnadze, JETP Lett. 56, 458 (1992).

[11] G. Volovik, J. Phys. Condens. Matter 5, 1759 (1993).

[12] I. Fomin, in Sov. Sci. Rev. Sec. A: Phys. Rev. (Harwood Acad. Publ., Chur, 1981), Vol. 3, pp. 305-308.

[13] I. Fomin, J. Low Temp. Phys. 31, 509 (1978).

[14] D. Osheroff and L. Corruccini, in Proceedings of the 14th International Conference on Low Temperature Physics (Elsevier, Amsterdam, 1975), Vol. 1, p. 100.

[15] A. Borovik-Romanov, Yu. Bunkov, V. Dmitriev, and Yu. Mukharskii, JETP Lett. 37, 716 (1983).

[16] V. Golo, A. Leman, and I. Fomin, JETP Lett. 38, 146 (1983). 